\title{
A Meta-Analysis of the Association between Diabetes Mellitus and Traditional Chinese Medicine Constitution
}

\author{
Fan Bai $\mathbb{D}^{1},{ }^{1}$ Hui Luo, ${ }^{1,2}$ Liying Wang, ${ }^{1}$ Linghui Zhu, ${ }^{1}$ Yuanyuan Guan, ${ }^{1}$ Yanfei Zheng $\mathbb{D}^{1}$ \\ Lingru Li $\mathbb{D}^{1}{ }^{1}$ and Qi Wang ${ }^{1}$ \\ ${ }^{1}$ Beijing University of Chinese Medicine, Beijing 100029, China \\ ${ }^{2}$ China Tibetology Research Center, Beijing 100101, China
}

Correspondence should be addressed to Yanfei Zheng; yanfei_z@163.com and Lingru Li; lilingru912@163.com

Received 30 April 2021; Accepted 22 July 2021; Published 4 August 2021

Academic Editor: Wen yi Kang

Copyright ( 92021 Fan Bai et al. This is an open access article distributed under the Creative Commons Attribution License, which permits unrestricted use, distribution, and reproduction in any medium, provided the original work is properly cited.

Objective. To explore the distribution of constitution types of diabetes mellitus (DM) in traditional Chinese medicine (TCM) and to provide evidence-based medicine basis for the prevention and treatment of diabetes. Methods. PubMed, Embase, Web of Science, and three Chinese databases were searched to include research literature on the relationship between diabetes and TCM constitution. The single rate study of cross-sectional literature was conducted with RStudio software, and the control metaanalysis of the diabetic and nondiabetic population was performed with Review Manager 5.3 software. Two independent reviewers assessed the methodological quality of the studies' data. The main outcomes included the distribution of constitutional types in the diabetic population and the odds ratio (OR) between the two. Effect sizes are expressed as proportions or ORs with $95 \%$ confidence intervals (CI). Results. A total of 28,781 diabetic cases were included in 87 articles. Yin-deficiency, phlegm-dampness, and qi-deficiency accounted for $18 \%$ (95\% CI (15\%, 20\%), $P<0.01), 17 \%(95 \% \mathrm{CI}(15 \%, 19 \%), P<0.01)$, and $13 \%$ (95\% CI (11\%, $15 \%), P<0.01)$ of the total diabetic cases. The risk of diabetes in people with yin-deficiency and phlegm-dampness was $3.06(95 \%$ CI (1.38-6.78), $P=0.006)$ and $1.89(95 \% \mathrm{CI}(1.05-3.42), P=0.03)$ times higher than that in those with other constitutions, respectively. The distribution of TCM constitution of DM patients varied significantly in different regions and ages. Conclusion. Yin-deficiency and phlegm-dampness are the common constitution types of diabetic people, and they may also be the risk factors of diabetes. Balanced constitution may be a protective factor of diabetes. More high-quality cohort and case-control studies need to be designed to provide more valuable evidence-based basis for assessing the correlation between DM and TCM constitution.

\section{Introduction}

Diabetes mellitus is a common chronic metabolic disease. With the development of society and the change of people's lifestyles, the incidence of DM has increased year by year, and it has become the most harmful noncommunicable disease on human health after tumor and cardiovascular disease [1]. According to the data released by the International T2DM Federation in 2013, there were 387 million diabetes patients in the world in 2013. China has the largest number of T2DM patients in the world, with 98 million T2DM patients, which is expected to increase to 143 million by 2035 [2]. The high incidence of chronic diseases and the rapid increase in medical costs highlight the importance of public health. In the face of the current high prevalence of diabetes, it is particularly important to carry out effective prevention and treatment measures. Traditional Chinese medicine (TCM) has been used for thousands of years of history as a form of health management, and this health care system has been widely used in many countries of the world [3]. TCM regards that everyone is different from others, so there is no recognized diagnosis and treatment model in the TCM treatment of diabetes, but more emphasis on individual treatment. Different constitution types make individuals suffer from different diseases. Individualized prevention and treatment measures for different types of constitution personalized treatment, so as to achieve a better effect than conventional treatment. The constitution of 
traditional Chinese medicine is the grasp of "preventive treatment of the disease" [4]. In April 2009, the Constitution in Chinese Medicine Questionnaire (CCMQ) was published and recommended by the China Association of Chinese Medicine as a standard for constitution measurement because of its good reliability and validity [5]. According to the CCMQ, the body constitutions of Chinese population are divided into nine basic types, namely balanced, qi-deficiency, yang-deficiency, yin-deficiency, phlegm-dampness, dampness-heat, blood stasis, qi stagnation, and inherited special. Among them, the balanced constitution is normal, while the other eight are biased and prone to relevant diseases [6]. TCM constitution identification has been widely used in nondisease treatment centers and community health service centers all over the country because of its simple operation, easy to understand, and easy to accept. In recent years, a large number of clinical studies on the relationship between diabetes and TCM constitution have been carried out in various regions, which provides data support for analyzing the distribution law of the TCM constitution of diabetic patients.

This study aims to obtain a larger sample of data on the distribution of TCM constitution types of diabetic patients, to determine the types of constitution prone to diabetes, and to provide evidence-based medical evidence for the effective prevention and treatment of diabetes for "preventive treatment of disease." This was achieved by a meta-analysis of the current clinical research literature on the TCM constitution of diabetes.

\section{Materials and Methods}

2.1. Retrieval Strategy. Clinical studies on the correlation between body constitution and diabetes were searched in PubMed, Embase, Web of Science, China National Knowledge Infrastructure (CNKI), VIP Database (VIP), and Wanfang Database from April 2009 (the CCMQ's publication time) to March 31, 2021. The search terms included "diabetes mellitus," "tangniaobing" (Chinese pinyin of diabetes), "tizhi" (Chinese pinyin of constitution), and "constitution." No language, nationality, or publication restrictions were applied. As an example of one specific strategy, the search terms for PubMed were as follows: (diabetes mellitus [Title/Abstract]) AND constitution [Title/ Abstract]).

2.2. Inclusion/Exclusion Criteria. All clinical literature on the relationship between diabetes and TCM constitution types (including cross-sectional studies, case-control studies, and cohort studies) were included, without limiting the type of research and the form of publication. The details are as follows: (1) Research object: the research object is the diabetic patients with a definite diagnosis; (2) Research tool: the measurement tool used for the constitution identification of the research object is the standard of Classification and Judgment of TCM Constitution issued by the China Institute of TCM in 2009; and (3) the sample size of the study is clear, and the data of the constitution composition are complete.
Exclusion criteria: (1) literature lacking in basic information reports or without statistics of constitution composition; (2) included subjects combined with other systematic serious diseases that may affect their TCM constitution types; (3) the study is limited to a certain kind of constitution population, such as simple phlegm-dampness constitution research; and (4) repeatedly published literature.

\subsection{Data Extraction and Quality Evaluation on Methodology.} Make a data extraction form, including the literature title, the type of research design, the area of the study, the sex of the object of study, the average age, the number of people of each type of constitution, the total sample size, and so on.

The Newcastle-Woodward scale (NOS) [7] was used to assess the methodological quality of the cohort study and case-control study. The scale was compared with 8 items from three aspects: the selection of the study population, the comparability between groups, and the measurement of exposure factors, with a total score of 9 points. The crosssectional study adopted the standard (referred to as AHRQ standard) recommended by the Agency for Healthcare Research and Quality (AHRQ) [8]. It is divided into 11 items, including data sources, inclusion criteria, observation time, continuity of research objects, subjective factors of evaluators, quality control, and so on, with a total score of 11 points.

2.4. Data Analysis. The meta-analysis of the individual rate of each constitution type was carried out by using RStudio1.1.463 software [9]. Analysis models were chosen by the results of heterogeneity tests. The percentage of 8 different biased constitution types of diabetic people and their $95 \%$ confidence interval (CI) were calculated. When the heterogeneity was too large, subgroup analysis was carried out according to regional and age factors, and the inverted funnel chart was used to analyze the degree of publication bias assessed by funnel plot analysis. The Review Manager 5.3 software provided by the Cochrane collaboration network was used to analyze the data synthesis between the diabetic group and nondiabetic group, and the odds ratio (OR) and its 95\% CI were used to describe the effect value of a single study. The effect model was selected according to heterogeneity: the fixed effect model was used when heterogeneity was less than $50 \%$, and the random effect model was used when heterogeneity was greater $\left(I^{2}>50 \%\right)$.

\section{Results}

3.1. Retrieval Process and Results. Duplicate references were excluded. All titles and abstracts were then screened to eliminate duplicates and obviously irrelevant citations. Following screening of 1240 citations found in the literature search, 93 papers potentially met the inclusion criteria and were examined in full text. 87 studies were included in the final review. The literature screening process and results are shown in Figure 1. 


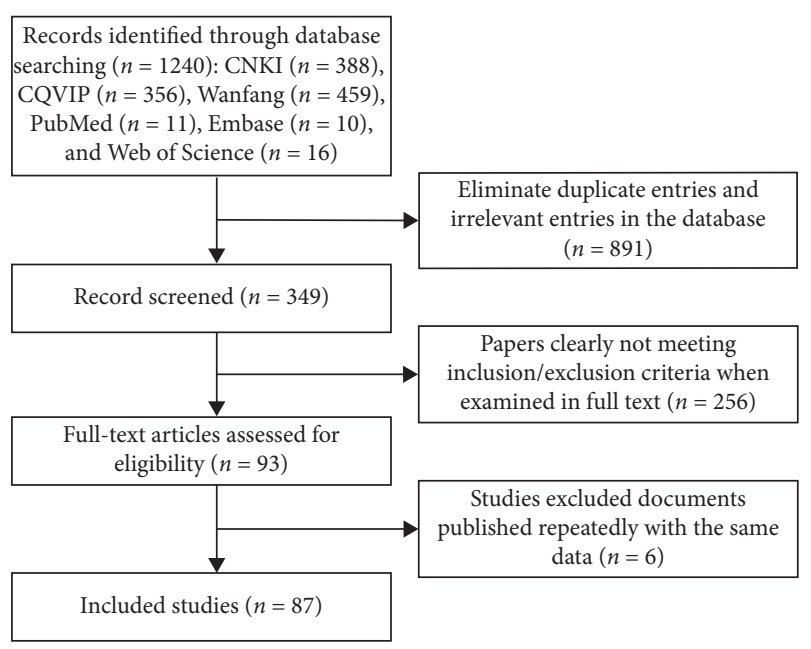

FIGURE 1: Flow chart of study searching and selection.

3.2. Basic Characteristics and Quality Evaluation of the Inclusion Study. Among all the studies we included, 1 was a Chinese-Malaysian constitution fitness study, and 86 were domestic clinical studies, including 22 provinces, autonomous regions, and municipalities directly under the central government. The study was published in 2009 at the earliest, and the number of studies is increasing year by year. A total of 28,581 patients with diabetes were included in each study, with an average sample size of 329 . According to the NOS (9 items) and AHRQ cross-sectional study evaluation criteria (11 items), 7 case-control studies and 80 cross-sectional studies were evaluated. The scores of cross-sectional studies were all 3-7, which were medium- and low-quality literatures, which did not have high research quality and had a high risk of bias. See Table 1 for details.

3.3. Meta-Analysis of Biased Constitution Distribution in TCM. According to the number of diabetic patients reported in each study, meta-analysis was carried out. A total of 87 studies reported these data, with a total sample size of 28,581 cases. The results showed that the distribution of 8 biased constitution types in patients with diabetes was yindeficiency, phlegm-dampness, and qi-deficiency in the highest proportion, and the results of meta-analysis were shown in a forest map. The proportion of the other five types of constitution is less than $10 \%$. The results of the metaanalysis are described in a table.

3.3.1. Yin-Deficiency Constitution. 86 studies involving 28,342 cases reported the proportion of yin-deficiency constitution in DM patient population. The random effects model was adopted due to the great heterogeneity of included studies. The results showed that the proportion of yin-deficiency constitution in DM patients was $18 \%$ (95\% CI (15\%, 20\%), $P<0.01$ ) (Figure 2).

3.3.2. Phlegm-Dampness Constitution. 86 studies involving 28,342 cases reported the proportion of phlegm-dampness constitution in DM patient population. The random effects model was adopted due to the great heterogeneity of included studies. The results showed that the proportion of phlegm-dampness constitution in DM patients was $17 \%$ (95\% CI $(15 \%, 19 \%), P<0.01)$ (Figure 3).

3.3.3. Qi-Deficiency Constitution. 84 studies involving 27,608 cases reported the proportion of qi-deficiency constitution in DM patient population. The random effects model was adopted due to the great heterogeneity of included studies. The results showed that the proportion of qideficiency constitution in DM patients was 13\% (95\% CI (11\%, 15\%), $P<0.01$ ) (Figure 4).

3.3.4. Other TCM Constitutions. The distribution proportion of the other five biased constitution in DM patients is less than $10 \%$, and the order from high to low is yangdeficiency, dampness-heat, blood stasis, qi stagnation, and inherited special constitution. The statistical results show that the $I^{2}$ values of all types of constitution are more than $50 \%$, suggesting that there is a large heterogeneity, so the random effect model should be used for meta-analysis (Table 2).

\subsection{Subgroup Analysis of Distribution of TCM Constitution by Region and Age}

3.4.1. Region. According to the regions included in the literature, all but one of the reports were conducted in China. Divided by the seven major regions of China, the majority of the literature included in this study is from North China, East China, and South China, with a large sample size, and other regions have smaller literature and smaller sample sizes. Therefore, all the literature was divided into three groups according to North China, East China, and South China to compare the proportions of yin-deficiency, phlegm-dampness, and qi-deficiency reported in the literature from the three regions. The results are shown in Table 3 .

3.4.2. Age. According to the average age of participants reported in each study, all the studies were divided into 3 subgroups by average age $(\leq 45,46-60$, and $>60)$. The random effects model was adopted due to the great heterogeneity of included studies. Meta-analysis showed that among the population of DM patients, the distribution of yin-deficiency, phlegm-dampness, and qi-deficiency constitution over 45 years old was significantly higher than that of adults under 45 years old (Table 4 ).

\subsection{Meta-Analysis of Distribution of TCM Constitution in DM} and General Population. The three most common types of constitution including yin-deficiency, phlegm-dampness, and qi-deficiency were identified in the meta-analysis of the proportion of constitution in DM patients. Then, we further compared it with general population. Seven studies [22, 35, 49, 50, 62, 73, 85] involving 36,546 participants 
TABLE 1: Characteristics of included studies.

\begin{tabular}{|c|c|c|c|c|c|c|}
\hline Study ID & Area & Study design & Sample size & Average age (years) & Gender ratio & Quality evaluation \\
\hline Li [10] & Jiangxi & CSS & 398 & $75.42 \pm 8.65$ & $206 / 192$ & 5 \\
\hline Zhong and Xue [11] & Guangdong & CSS & 50 & $62.99 \pm 3.49$ & $25 / 25$ & 6 \\
\hline Liu et al. [12] & Henan & CSS & 109 & $57.94 \pm 8.69$ & $58 / 51$ & 4 \\
\hline Zhang et al. [13] & Shanghai & CSS & 120 & $56.88 \pm 2.42$ & $65 / 55$ & 4 \\
\hline $\mathrm{Wu}$ et al. [14] & Ningxia & CSS & 100 & $41.6 \pm 4.5$ & $68 / 32$ & 5 \\
\hline Wang et al. [15] & Shanghai & CSS & 112 & $55.8 \pm 6.2$ & $54 / 58$ & 5 \\
\hline Shen et al. [16] & Shanghai & CSS & 395 & 73.81 & $172 / 223$ & 4 \\
\hline Lin et al. [17] & Guangdong & CSS & 500 & $61.2 \pm 5.3$ & $258 / 242$ & 6 \\
\hline Li et al. [18] & Guizhou & CSS & 663 & $59.12 \pm 6.57$ & $411 / 252$ & 3 \\
\hline Hang et al. [19] & Guangdong & CSS & 250 & $71.01 \pm 5.82$ & $133 / 117$ & 4 \\
\hline Guan $[20]$ & Jiangsu & CSS & 200 & $\mathrm{U}$ & $105 / 95$ & 3 \\
\hline Guan [21] & Sichuan & CSS & 195 & $69.36 \pm 3.41$ & $109 / 86$ & 7 \\
\hline Zhang et al. [22] & Zhejiang & CCS & 1315 & U & $890 / 425$ & 6 \\
\hline Zhou [23] & Fujian & CCS & 285 & $56.98 \pm 6.75$ & $\mathrm{U}$ & 5 \\
\hline Wei and Yang [24] & Guangdong & CSS & 300 & U & $\mathrm{U}$ & 5 \\
\hline Wang [25] & Neimongol & CSS & 270 & $66.8 \pm 10.5$ & $136 / 134$ & 6 \\
\hline Lin et al. [26] & Beijing & CSS & 122 & U & U & 4 \\
\hline Li et al. [27] & Beijing & CSS & 119 & $\mathrm{U}$ & $71 / 48$ & 5 \\
\hline Lang [28] & Heilongjiang & CSS & 100 & $75.12 \pm 6.34$ & $56 / 44$ & 7 \\
\hline Jiang et al. [29] & Shanghai & CSS & 700 & $60.14 \pm 4.55$ & $350 / 350$ & 5 \\
\hline Hu et al. [30] & Tianjin & CSS & 200 & $61.32 \pm 8.24$ & $96 / 104$ & 5 \\
\hline Gao et al. [31] & Shandong & CSS & 75 & 69.92 & $33 / 42$ & 4 \\
\hline Chen et al. [32] & Zhejiang & CSS & 700 & $74.1 \pm 13.1$ & $242 / 458$ & 5 \\
\hline Zhou et al. [33] & Shanghai & CSS & 442 & $\mathrm{U}$ & $187 / 255$ & 7 \\
\hline Wang [34] & Shandong & CSS & 436 & $58.12 \pm 9.63$ & $255 / 181$ & 7 \\
\hline Shen et al. [35] & Beijing & CCS & 461 & $70.68 \pm 10.41$ & $202 / 259$ & 6 \\
\hline Shen et al. [36] & Beijing & CSS & 333 & $57.80 \pm 10.91$ & $133 / 200$ & 6 \\
\hline Qiu [37] & Xinjiang & CSS & 141 & $61.89 \pm 11.37$ & $86 / 55$ & 5 \\
\hline Lv [38] & Tianjin & CSS & 1003 & $\mathrm{U}$ & U & 7 \\
\hline Liu et al. [39] & Jiangxi & CSS & 208 & $52.1 \pm 3.4$ & $107 / 91$ & 5 \\
\hline $\mathrm{Li}[40]$ & Beijing & CSS & 60 & $\mathrm{U}$ & $31 / 29$ & 4 \\
\hline Jing et al. [41] & Beijing & CSS & 160 & $56.35 \pm 12.82$ & $74 / 86$ & 4 \\
\hline Jing [42] & Guangxi & CSS & 297 & $57.59 \pm 11.12$ & $176 / 121$ & 5 \\
\hline Huang [43] & Guangdong & CSS & 497 & $67.94 \pm 8.64$ & $200 / 297$ & 4 \\
\hline Huang et al. [44] & Guangdong & CSS & 367 & $67.57 \pm 8.77$ & $137 / 230$ & 4 \\
\hline Huang et al. [45] & Zhejiang & CSS & 630 & $\mathrm{U}$ & $236 / 394$ & 4 \\
\hline Zhu et al. [46] & Nationwide & CSS & 412 & $52.54 \pm 13.92$ & $\mathrm{U}$ & 7 \\
\hline Feng [47] & Jiangsu & CSS & 200 & 61.4 & $103 / 97$ & 7 \\
\hline Zou and Wang [48] & Sichuan & CSS & 320 & $52.11 \pm 11.32$ & $167 / 153$ & 4 \\
\hline Zhu et al. [49] & Beijing & CSS & 136 & $\mathrm{U}$ & $\mathrm{U}$ & 4 \\
\hline Xue et al. [50] & Shanghai & CCS & 879 & $\mathrm{U}$ & $425 / 454$ & 5 \\
\hline $\mathrm{Xu}$ and Liu [51] & Shanxi & CSS & 251 & 59.666 & $114 / 133$ & 4 \\
\hline Xie et al. [52] & Henan & CSS & 426 & $\mathrm{U}$ & $258 / 198$ & 5 \\
\hline Wang et al. [53] & Guangdong & CSS & 112 & $86.61 \pm 13.39$ & $45 / 67$ & 5 \\
\hline Wang et al. [54] & Jiangsu & CSS & 300 & $\mathrm{U}$ & $144 / 156$ & 6 \\
\hline Wang [55] & Sichuan & CSS & 57 & $39.04 \pm 12.09$ & $10 / 17$ & 7 \\
\hline Ren et al. [56] & Shandong & CSS & 151 & 63.8 & $83 / 68$ & 6 \\
\hline $\mathrm{Li}[57]$ & Guangdong & CSS & 120 & $51.9 \pm 4.7$ & $68 / 52$ & 5 \\
\hline Li et al. [58] & Guangxi & CSS & 302 & $65.60 \pm 10.96$ & $130 / 172$ & 6 \\
\hline Huang [59] & Malaysia & CSS & 68 & $64.16 \pm 10.03$ & $30 / 38$ & 5 \\
\hline Hua et al. [60] & Shanghai & CSS & 931 & $73.45 \pm 7.37$ & $353 / 578$ & 4 \\
\hline Zou and Wang [61] & Beijing & CSS & 296 & $49.70 \pm 12.28$ & $173 / 123$ & 4 \\
\hline Zhao [62] & Inner Mongolia & CCS & 139 & $\mathrm{U}$ & $84 / 55$ & 7 \\
\hline Zhang [63] & Henan & CSS & 90 & 40 & $71 / 19$ & 5 \\
\hline $\mathrm{Yu}[64]$ & Liaoning & CSS & 372 & $60.91 \pm 11.557$ & $173 / 199$ & 6 \\
\hline Yang [65] & Shandong & CSS & 192 & $63.4 \pm 8.0$ & $80 / 112$ & 7 \\
\hline Xiang and Qian [66] & Jiangsu & CSS & 835 & 63.4 & $343 / 492$ & 5 \\
\hline Wu and Hao [67] & Shanxi & CSS & 354 & $\mathrm{U}$ & $196 / 158$ & 5 \\
\hline Wu et al. [68] & Fujian & CSS & 476 & $\mathrm{U}$ & $188 / 288$ & 4 \\
\hline Wei et al. [69] & Shanghai & CSS & 400 & $59.8 \pm 11.6$ & $211 / 189$ & 6 \\
\hline
\end{tabular}


TABLE 1: Continued.

\begin{tabular}{|c|c|c|c|c|c|c|}
\hline Study ID & Area & Study design & Sample size & Average age (years) & Gender ratio & Quality evaluation \\
\hline Wang et al. [70] & Guangdong & CSS & 320 & 47.2 & $175 / 145$ & 5 \\
\hline $\mathrm{Lu}[71]$ & Shanghai & CSS & 1420 & 69.2 & $574 / 846$ & 3 \\
\hline Liu et al. [72] & Tianjin & CSS & 200 & $\mathrm{U}$ & $\mathrm{U}$ & 4 \\
\hline $\mathrm{Li}[73]$ & Guangdong & CCS & 483 & $42.03 \pm 12.68$ & $263 / 220$ & 6 \\
\hline $\mathrm{Li}[74]$ & Fujian & CSS & 250 & $70.12 \pm 11.92$ & $204 / 261$ & 5 \\
\hline $\mathrm{Hu}$ et al. [75] & Jiangxi & CSS & 500 & $57.1 \pm 11.4$ & $188 / 312$ & 6 \\
\hline Chen and Zhou [76] & Zhejiang & CSS & 119 & 57.6 & $65 / 54$ & 6 \\
\hline Xiao et al. [77] & Guangdong & CSS & 300 & $65.6 \pm 12.6$ & $160 / 140$ & 5 \\
\hline Zhu et al. [78] & Shandong & CSS & 135 & $55.69 \pm 9.87$ & $56 / 79$ & 6 \\
\hline Yang and Wang [79] & Yunnan & CSS & 427 & $\mathrm{U}$ & $\mathrm{U}$ & 6 \\
\hline Xiang and Ran [80] & Jiangsu & CSS & 270 & $\mathrm{U}$ & $116 / 154$ & 5 \\
\hline Xiang [81] & Jiangsu & CSS & 478 & $63.1 \pm 9.1$ & $201 / 277$ & 5 \\
\hline Wang et al. [82] & Beijing & CSS & 213 & $56.55 \pm 12.14$ & $99 / 104$ & 6 \\
\hline Li et al. [83] & Zhejiang & CSS & 300 & $56.57 \pm 7.91$ & $152 / 148$ & 5 \\
\hline Chen et al. [84] & Henan & CSS & 300 & $52.12 \pm 12.19$ & $152 / 148$ & 5 \\
\hline Zheng and Jian [85] & Guangdong & CCS & 89 & $\mathrm{U}$ & $54 / 35$ & 6 \\
\hline Wu et al.[86] & Guangdong & CSS & 147 & $\mathrm{U}$ & $\mathrm{U}$ & 6 \\
\hline Tang [87] & Guangxi & CSS & 200 & $52.78 \pm 6.62$ & $99 / 101$ & 3 \\
\hline $\mathrm{Ma}[88]$ & Guangdong & CSS & 48 & $56.9 \pm 6.7$ & $35 / 13$ & 4 \\
\hline Liu [89] & Guangdong & CSS & 98 & $56.6 \pm 4.8$ & $55 / 43$ & 6 \\
\hline Li et al. [90] & Guangdong & CSS & 74 & 62.6 & $39 / 35$ & 5 \\
\hline Zhang et al. [91] & Hong Kong & CSS & 239 & $59 \pm 10.33$ & $119 / 120$ & 5 \\
\hline Han [92] & Beijing & CSS & 206 & $65 \pm 12$ & $91 / 115$ & 6 \\
\hline Han et al. [93] & Beijing & CSS & 52 & $58.14 \pm 8.2$ & $41 / 11$ & 4 \\
\hline Zhang [94] & Fujian & CSS & 534 & $57.67 \pm 11.17$ & $258 / 276$ & 5 \\
\hline Yan et al. [95] & Henan & CSS & 571 & $51.69 \pm 8.81$ & $284 / 287$ & 6 \\
\hline Cui $[96]$ & Shanxi & CSS & 476 & U & $188 / 288$ & 4 \\
\hline
\end{tabular}

CSS, cross-sectional study; CCS, case-control study; U, unclear, indicating no report.

reported the relevant data, so they were included in the meta-analysis.

Meta-analysis showed that the OR value of yin-deficiency, phlegm-dampness, and balanced constitution's distribution in DM patients and general population was 3.06 (95\%CI: 1.38-6.78), 1.89 (95\%CI: 1.05-3.42), and 0.48 (95\% CI: $0.32-0.71)$, respectively. The difference was statistically significant. There was no significant difference in the proportion of qi-deficiency between the DM and general populations, with an OR of 1.82 (95\%CI: 0.68-4.81). More details are shown in Figure 5.

3.6. Publication Bias. Taking the proportion of yin-deficiency constitution of participants with DM as an index, the inverted funnel diagram was analyzed in the included literature. Considering that TCM constitution research is mostly carried out in different regions and people, and the differences in climate, environment, and lifestyle lead to obvious heterogeneity of each research, so it presents a relatively scattered state, which is related to the particularity of TCM constitution research (Figure 6).

\section{Discussion}

4.1. Analysis of the Correlation between DM and the Constitution of TCM. Prevention is the one of the most important essences of TCM, indicating the preventive treatment of diseases. The body constitution in TCM could indicate the patients' overall condition and could form the basis of prevention theory; thus, it has attracted much attention from researchers and clinicians [97]. The TCM constitution refers to the comprehensive, relatively stable, and inherent characteristics of the morphological structure, physiological function, and psychological state formed on the basis of innate endowment and acquired disposition in the course of human life [98]. It can reflect an individual's current health status and future health trends in constitution differences, life processes, psychological condition, and adaptability to natural and social environments. The specificity of individual constitution often causes body's susceptibility to a specific pathogenic factor. For instance, people with phlegmdampness constitution easily suffer from disease such as diabetes mellitus, metabolic syndrome, and other diseases, with weak adaptability to rainy season and moist environment [99]. The TCM constitution scale provided an objective, generally recognized technical tool and professional standard. Through the constitution classification of TCM, we can determine the individual constitution type of the population and guide the individualized treatment and disease prevention. Correlative studies on constitution and clinical disease have promoted the application of TCM in public health. Relevant research shows that yin-deficiency constitution of hypertension, diabetes, constipation, female menopause, and osteoporosis showed high distribution [100]. This is also consistent with our research results.

A total of 87 articles were included in this study, with a total of 28,781 large samples. It was found that yin- 


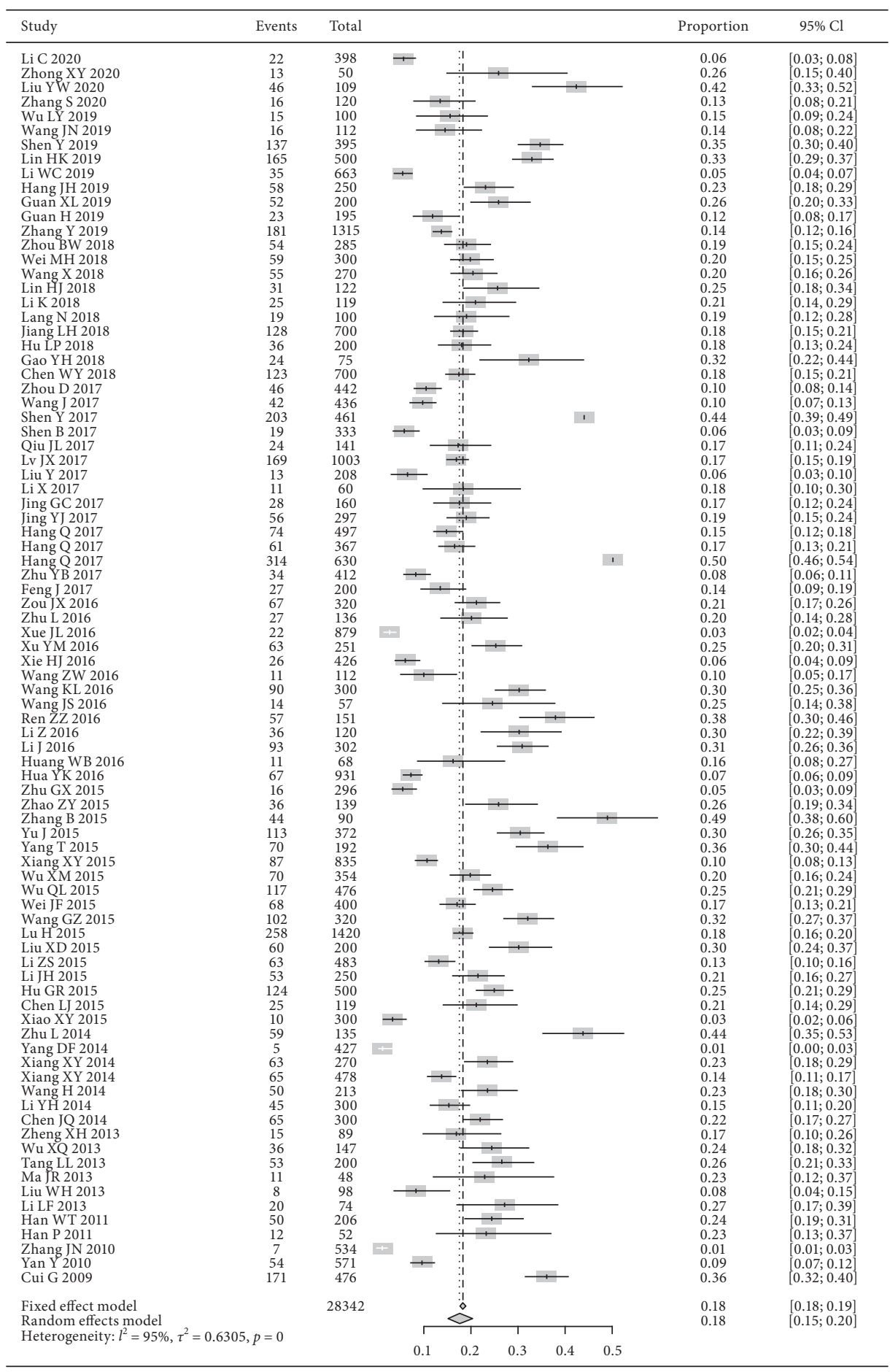

Figure 2: Meta-analysis of the proportion of yin-deficiency constitution in DM patients.

deficiency, phlegm-dampness, and qi-deficiency constitution accounted for $18 \%(15 \%-20 \%), 17 \%(15 \%-19 \%)$, and $13 \%(11 \%-15 \%)$ of the total number of people with diabetes, respectively. The results of meta-analysis showed that balanced constitution may be a protective factor of diabetes $(\mathrm{OR}=0.48,95 \% \mathrm{CI}: 0.32-0.71)$, while yin-deficiency and phlegm-dampness constitution may be the risky factors of it $(\mathrm{OR}=3.06,95 \% \mathrm{CI}: 1.38-2.78$ and $\mathrm{OR}=1.89,95 \% \mathrm{CI}$ : $1.05-3.42)$.
The constitution distribution of DM patients was significantly different from that of the general population. In 2009, Zhu and Wang published a nationwide cross-sectional study [101] on the constitution of 21,948 general populations in TCM. The results showed that balanced constitution (32.14\%) was the most common type of TCM constitution. Qi-deficiency (13.42\%), yang-deficiency (9.08\%), and phlegm-dampness $(9.04 \%)$ were common biased constitution. In this study, yin-deficiency constitution was the most 


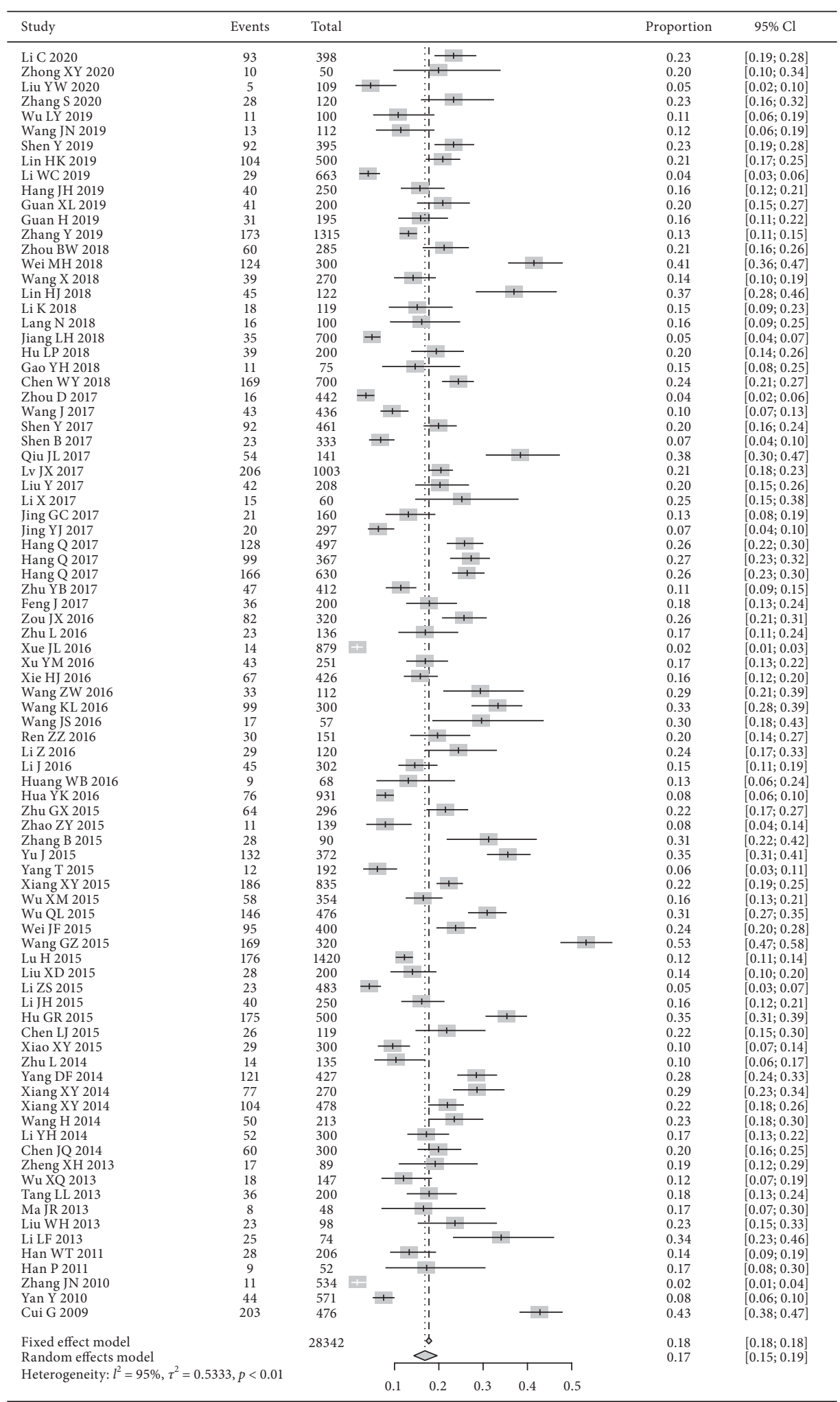

FIGURE 3: Meta-analysis of the proportion of phlegm-dampness constitution in DM patients.

common (18\%) followed by phlegm-dampness (17\%) and qideficiency $(13 \%)$. The proportion of yin-deficiency and phlegm-dampness constitution is much higher than that in general population, while qi-deficiency was close to that of general population. This could illustrate the specificity of constitution characteristics in patients with DM and may also confirm the association between DM and TCM constitution. It is suggested that in the process of prevention and 


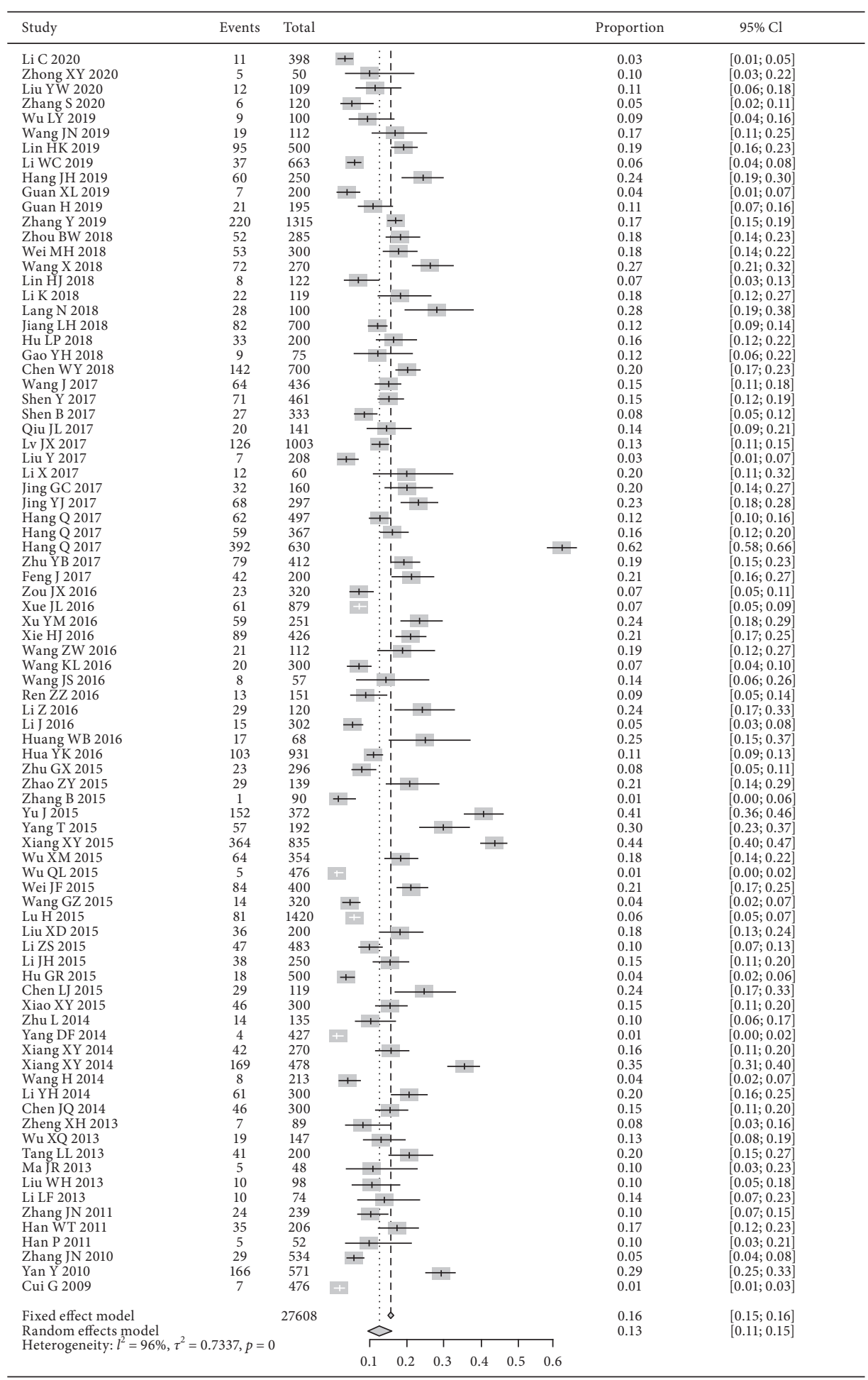

FIgURE 4: Meta-analysis of the proportion of qi-deficiency constitution in DM patients.

TABLE 2: Meta-analysis of the proportion of other five constitutions in DM patients.

\begin{tabular}{lcccccc}
\hline Constitution & Studies & Participants & Proportion (\%) & $95 \%$ CI & $P$ & $I^{2}(\%)$ \\
\hline Yang-deficiency & 79 & 26025 & 8 & $7 \%-9 \%$ & $<0.01$ \\
Dampness-heat & 78 & 26209 & 7 & $6 \%-9 \%$ & $<0.01$ \\
Blood stasis & 83 & 27290 & 6 & $5 \%-7 \%$ & $<0.01$ \\
Qi stagnation & 73 & 24692 & 4 & $3 \%-5 \%$ & $<0.01$ \\
Inherited special & 62 & 21295 & 2 & $1 \%-2 \%$ & $<0$ \\
\hline
\end{tabular}


TABLE 3: Meta-analysis of common constitution proportion of DM in three regions.

\begin{tabular}{|c|c|c|c|c|c|c|c|}
\hline Constitution & Region & Studies & Number of occurrence & Total sample size & Proportion (\%) & $95 \% \mathrm{CI}$ & $P$ \\
\hline \multirow{3}{*}{ Yin-deficiency } & East China & 32 & 2472 & 14386 & 16 & $13 \%-20 \%$ & $<0.01$ \\
\hline & South China & 20 & 944 & 4793 & 17 & $13 \%-21 \%$ & $<0.01$ \\
\hline & North China & 18 & 961 & 4575 & 20 & $16 \%-25 \%$ & $<0.01$ \\
\hline \multirow{3}{*}{ Phlegm-dampness } & East China & 32 & 2351 & 14386 & 15 & $12 \%-19 \%$ & $<0.01$ \\
\hline & South China & 19 & 980 & 4554 & 20 & $15 \%-26 \%$ & $<0.01$ \\
\hline & North China & 18 & 812 & 4575 & 17 & $14 \%-20 \%$ & $<0.01$ \\
\hline \multirow{3}{*}{ Qi-deficiency } & East China & 31 & 2241 & 13991 & 11 & $7 \%-16 \%$ & $<0.01$ \\
\hline & South China & 20 & 690 & 4793 & 13 & $11 \%-16 \%$ & $<0.01$ \\
\hline & North China & 18 & 662 & 4575 & 13 & $9 \%-17 \%$ & $<0.01$ \\
\hline
\end{tabular}

TABLe 4: Meta-analysis of distribution of TCM constitutions by age in DM patients.

\begin{tabular}{|c|c|c|c|c|c|c|}
\hline Constitution & Age & Studies & Participants & Proportion (\%) & $95 \% \mathrm{CI}$ & $P$ \\
\hline \multirow{3}{*}{ Yin-deficiency } & $\leq 45$ & 4 & 136 & 23 & $12 \%-38 \%$ & $<0.01$ \\
\hline & $46-60$ & 31 & 1352 & 14 & $11 \%-19 \%$ & $<0.01$ \\
\hline & $>60$ & 29 & 1916 & 19 & $15 \%-23 \%$ & $<0.01$ \\
\hline \multirow{3}{*}{ Phlegm-dampness } & $\leq 45$ & 4 & 79 & 15 & $7 \%-31 \%$ & $<0.01$ \\
\hline & $46-60$ & 30 & 1356 & 15 & $11 \%-19 \%$ & $<0.01$ \\
\hline & $>60$ & 29 & 1846 & 18 & $16 \%-21 \%$ & $<0.01$ \\
\hline \multirow{3}{*}{ Qi-deficiency } & $\leq 45$ & 4 & 65 & 8 & $4 \%-14 \%$ & $\leq 0.01$ \\
\hline & $46-60$ & 31 & 1173 & 12 & $9 \%-15 \%$ & $<0.01$ \\
\hline & $>60$ & 28 & 1699 & 16 & $13 \%-20 \%$ & $<0.01$ \\
\hline
\end{tabular}

treatment of diabetes in the future, we should identify and focus on the people with yin-deficiency and phlegmdampness constitution as soon as possible and take corresponding intervention measures in exercise, diet, and so on, to promote the return of biased constitution to balanced constitution.

The results of meta-analysis showed that the proportion of yin-deficiency was the highest in North China followed by South and East China, and the proportion of phlegmdampness was the highest in South China followed by North China and East China, which may be related to the climatic characteristics of different regions. The climate in North China is cool and dry, the humidity of the air is insufficient, and the body fluid is deficient and dry, so that there are more people with yin-deficiency. The climate in South China is warm and humid, which may easily generate phlegm, dampness, and heat inside the bodies of local residents, which may lead to more people with phlegm-dampness. East China runs through the north and south areas, and the climate contains dry and wet, so people with yin-deficiency and phlegm-dampness account for more. The age subgroup showed that the number of people with diabetes over 45 years old was significantly more than that under 45 years old. Some studies have shown that age is one of the most important factors affecting diabetes [102], advanced age is a risk factor for diabetes, and the prevalence rate of diabetes increases with age. Hence, efforts should be made to adjust the constitution in middle-aged and elderly populations about diabetes mellitus to reduce the incidence of type 2 diabetes.
4.2. Limitations of the Study. From the perspective of the methodology of research design, the literature reports on the basic information related to the research is incomplete, such as the time of inclusion of the research object, basic data, and the original data of each constitution. In the aspect of data statistical analysis, some studies only describe the number and proportion of various constitution types and there is a lack of research on the correlation between constitution types and family heredity, external environment, illness and other factors.

The causes of diabetes are complex, but due to the lack of reported data in the original literature, the analysis angle has some limitations, so it is difficult to deeply analyze the various factors that affect the constitution of people with diabetes. This study is different from other types of metaanalysis. The articles included are mainly observational studies, which can only hint but not prove the causal relationship between disease and constitution. The results of this study need to be confirmed by more high-quality prospective cohort studies and case-control studies. Therefore, there may be a certain risk of deviation in the result.

The included studies are heterogeneous. Due to the large amount of literature, there are significant differences in research time, measurement methods, sample size, age, gender, region, and other aspects. Different from intervention research, individual differences have a great impact on TCM constitution, which is also the characteristic of TCM constitution. Therefore, this study conducted 


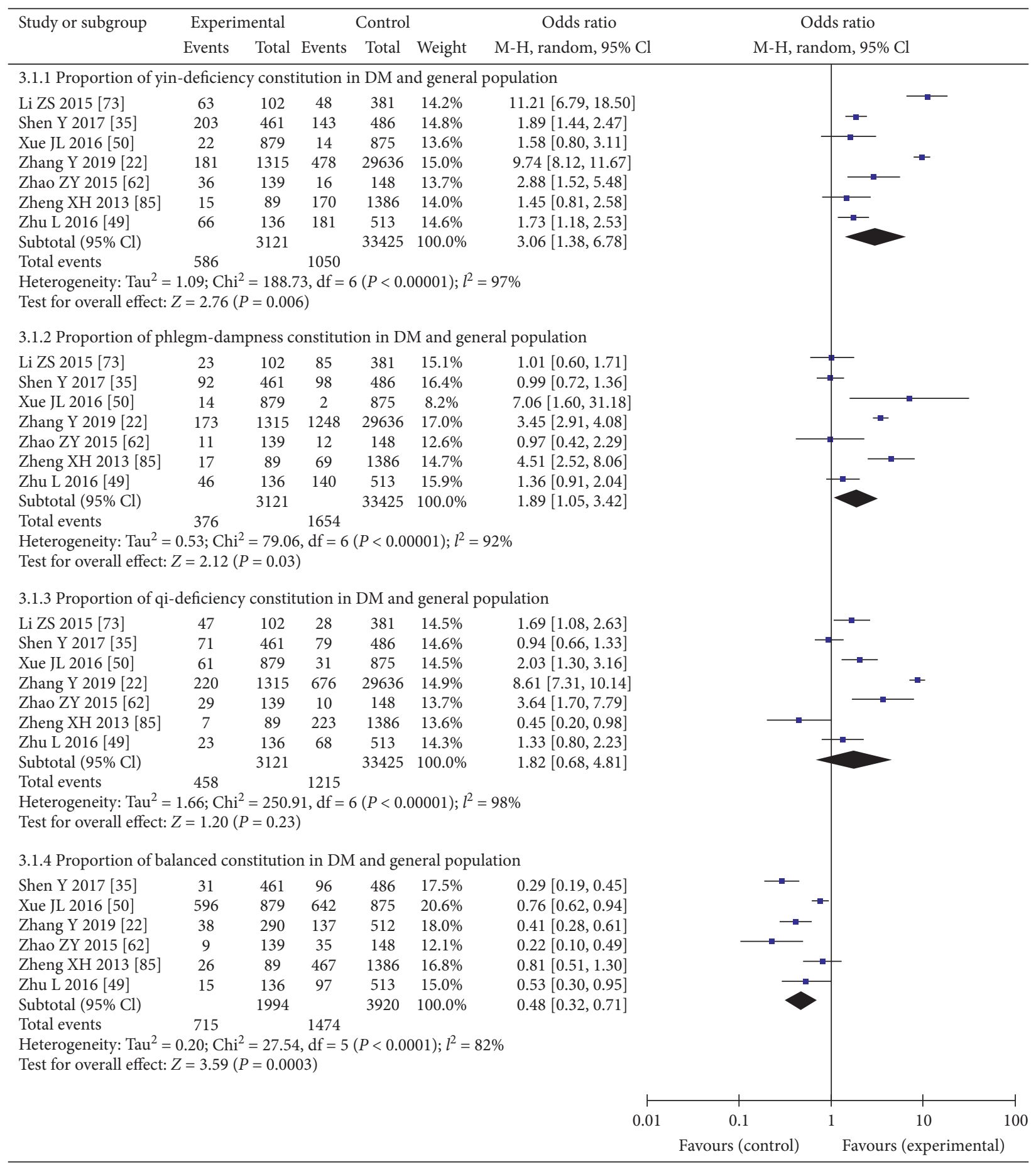

FIgUre 5: Comparison of distribution of three common traditional Chinese medicine constitutions and balanced constitution between DM patients and general population.

subgroup analyses of the original data by region and age in order to better understand. It is suggested that clinical research methods with higher quality and more standardized contents should be adopted in the future to further explore the relationship between various TCM constitutions and chronic diseases.
4.3. Implications for Future Clinical Practice. Referring to the methods of evidence-based medicine in the previous study [103] on the relationship between constitution and disease, this study analyzed the people with high incidence of diabetes from the perspective of TCM constitution. Combined with published studies [103, 104], we have found that 


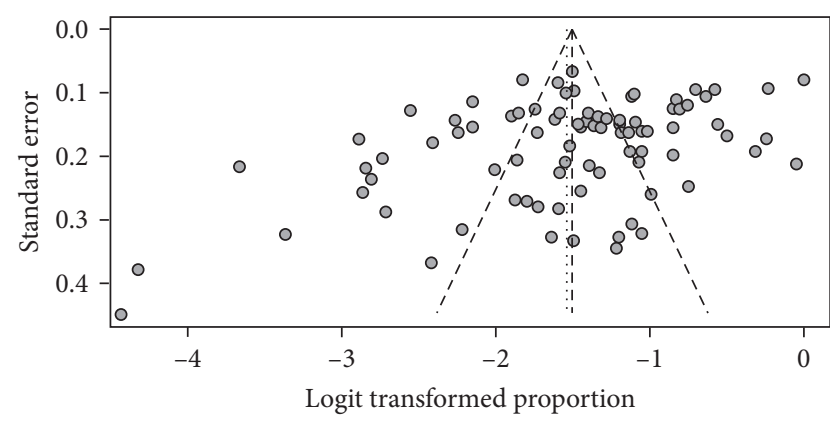

FIGURE 6: Funnel plot analysis of the distribution of yin-deficiency constitution.

phlegm-dampness constitution is closely related to various metabolic diseases, which is of great significance to guide the accurate prevention and treatment of metabolic diseases. In the future clinical practice, we can determine the target of early intervention of diabetes by screening the high-risk groups of yin-deficiency and phlegm-dampness and carry out targeted drug and nondrug intervention according to the constitution and syndrome of TCM, in order to adjust biased constitution and reduce the risk of disease.

\section{Conclusion}

The current systematic review of 87 included studies found that yin-deficiency, phlegm-dampness, and qi-deficiency are the main types of constitution in diabetes patients, of which yin-deficiency and phlegm-dampness are associated with the occurrence of diabetes. They are also risk factors for the incidence of diabetes, while balanced constitution is a protective factor for diabetes. It is suggested that in the future, we should focus on people over 45 years old with yindeficiency in North China and phlegm-dampness people over 45 years old in South China and increase TCM constitution intervention measures as soon as possible to improve biased constitution and reduce the incidence of diseases. More high-quality observational studies should be designed in the future to provide a more scientific evidencebased basis for the use of personalized diagnosis and treatment of TCM in the prevention and treatment of diabetes.

\section{Data Availability}

The relevant data used to support the results of this study are included in this article.

\section{Conflicts of Interest}

The authors declare that there are no conflicts of interest regarding the publication of this paper.

\section{Authors' Contributions}

Fan Bai and Hui Luo contributed equally to this work. Fan Bai and Hui Luo put forward the idea of this study and designed the method of this systematic review. Fan Bai,
Liying Wang, Linghui Zhu, and Yuanyuan Guan searched the articles, conducted data collection and extraction, and analyzed the data. Lingru Li, Yanfei Zheng, and Qi Wang provided guidelines for this systematic review and metaanalysis. The authors approved the final version of the manuscript.

\section{Acknowledgments}

This research was financially supported by the National Natural Science Foundation of China (no. 81874413), Science and Technology Nova Plan of Beijing City (no. Z201100000820027), Young Talent Promotion Project of China Association of Chinese Medicine (no. CACM-2018QNRC2-C02), and Youth Innovation Team Project of Beijing University of Traditional Chinese Medicine (no. 2019-JYB-TD-010).

\section{References}

[1] L. Yu, N. W. Liu, L. Zhang et al., "An epidemiological survey of diabetes mellitus and prediabetes in adults in Tianjin," Chinese Journal of Prevention and Control of Chronic Diseases, vol. 25, no. 7, pp. 511-513, 2017.

[2] L. Guariguata, D. R. Whiting, I. Hambleton et al., "Global estimates of diabetes prevalence for 2013 and projections for 2035," Diabetes Research and Clinical Practice, vol. 103, pp. 137-149, 2017.

[3] L. Li, H. Yao, J. Wang, Y. Li, and Q Wang, "The role of Chinese medicine in health maintenance and disease prevention: application of constitution theory," The American Journal of Chinese Medicine, vol. 47, no. 3, pp. 495-506, 2019.

[4] Q. Wang, "Physical identification is "Treat the disease" of 'Gripper'," Global Traditional Chinese Medicine, vol. 4, no. 3, pp. 5-6, 2008.

[5] Y. B. Zhu, Q. Wang, and H. Origasa, "Evaluation on reliability and validity of the constitution in Chinese medicine questionnaire (CCMQ)," Chinese Journal of Behavioral Medicine Science, vol. 16, no. 7, pp. 651-654, 2007.

[6] Q. Wang, "Individualized medicine, health medicine, and constitutional theory in Chinese medicine," Frontiers of Medicine, vol. 6, no. 1, pp. 1-7, 2012.

[7] G. Wells, B. Shea, D. O'Connell, and J. Peterson, The Newcastle-Ottawa Scale (NOS) for Assessing the Quality of Non-randomized Studies in Meta-Analysis, Ottawa Hospital Research Institute, Ottawa, Canada, 2000.

[8] A. Rostom, C. Dube, and A. Cranney, Summary, Evidence Report/technology Assessment No 104 (Prepared by the University of Ottawa Evidence-Based Practice Center, under contract, No. 290-02-0021), 2004.

[9] M. L. Luo, H. Z. Tan, and Q. Zhou, "Realizing the metaanalysis of single rate in R," The Journal of Evidence-Based Medicine, vol. 13, pp. 181-184, 2013.

[10] C. Li, "Identification and analysis of TCM constitution of diabetic population in lujiang community of zhangshu city," The Medical Forum, vol. 24, no. 31, pp. 4579-4580, 2020.

[11] X. Y. Zhong and L. Xue, "Effects of TCM biased constitution on type 2 diabetes mellitus and impaired glucose regulation," Chinese and Foreign Medical Research, vol. 18, no. 16, pp. 126-127, 2020.

[12] Y. W. Liu, K. W. Zhang, H. Y. Ji et al., "Correlation between the constitution of type 2 diabetes mellitus and the twelve meridians was discussed based on palmar meridians 
detection instrument," Henan Traditional Chinese Medicine, vol. 40, no. 1, pp. 120-122, 2020.

[13] S. Zhang, S. X. Zhang, Y. Ling et al., "Investigation of traditional Chinese medicine constitution types in 120 patients with type 2 diabetes and comparison of different constitution-related glucose metabolism indexes," Shanghai Journal of Traditional Chinese Medicine, vol. 54, no. 1, pp. 7-10, 2020.

[14] L. Y. Wu, W. Liu, and X. X. Liu, "The intervention study of TCM constitution identification on hypertension and type 2 diabetes mellitus," Nei Mongol Journal of Traditional Chinese Medicine, vol. 38, no. 8, pp. 77-80, 2019.

[15] J. N. Wang, L. Y. Du, Y. W. Zhao et al., "Correlation and mechanism between constitution in traditional Chinese medicine and mild cognitive impairment of patients with type 2 diabetes mellitus," Shandong Journal of Traditional Chinese Medicine, vol. 38, no. 11, pp. 1031-1036, 2019.

[16] Y. Shen, H. Tang, D. Zhou et al., "Association of TCM constitution with gene polymorphisms of interleukin-6, interleukin-10, and interleukin-10 receptor 1 in patients with type 2 diabetes," Journal of Anhui University of Chinese Medicine, vol. 38, no. 3, pp. 6-11, 2019.

[17] H. K. Lin, W. F. Huang, W. B. Zhan et al., "Investigation on the constitution type of Chinese medicine in middle-aged and old patients with type 2 diabetes," Shenzhen Journal of Integrated Traditional Chinese and Western Medicine, vol. 29, no. 8, pp. 59-60, 2019.

[18] W. C. Li, J. Feng, and L. L. Liu, "Analysis of constitution identification of type 2 diabetes mellitus," Yiyaojie, vol. 6, p. 40, 2019.

[19] J. H. Huang, W. X. Zhu, Q. Xia et al., "Study on the relationship between sympathetic activity of autonomic nervous system and vagal activity, their balances and coordination in patients with diabetes mellitus with different constitutions of traditional Chinese medicine," Guangzhou Medical Journal, vol. 3, pp. 84-87, 2019.

[20] X. L. Guan, "Analysis and discussion on constitution of traditional Chinese medicine in community patients with type 2 diabetes mellitus," Journal of Community Medicine, vol. 17, no. 9, pp. 529-531, 2019.

[21] H. Guan, The Implementation and Effect Evaluation of Constitution Intervention in the Health Management of Type 2 Diabetes Mellitus, Southwest Medical University, Luzhou, China, 2019.

[22] Y. Zhang, Y. Y. Zhang, R. W. Yang et al., "Establishment and verification of diabetes risk assessment model based on TCM constitution identification," Chinese General Practice, vol. 23, no. 637, pp. 68-73, 2020.

[23] B. W. Zhou, Study on the Relationship between the Constitution Type and the Main Risk Factors of Cardiovascular System in Type 2 Diabetic Patients, Fujian University of traditional Chinese Medicine, Fujian, China, 2018.

[24] M. H. Wei and Y. J. Yang, "Investigation on the constitution of traditional Chinese medicine of type 2 diabetes mellitus and prediabetes at Panyu District, Guangzhou," International Medicine and Health Guidance News, vol. 24, no. 4, pp. 480-482, 2018.

[25] X. Wang, "Relationship between body type and correlation index of diabetic patients in Baotou area," Journal of Baotou Medical College, vol. 34, no. 6, pp. 85-87, 2018.

[26] H. J. Lin, Y. Jiang, Y. J. Liu et al., "Influence of family health guidance on blood glucose of diabetic patients based on constitution identification of traditional Chinese Medicine," Guide of China Medicine, vol. 16, no. 16, pp. 196-197, 2018.
[27] K. Li, Y. X. Lin, L. R. Ma et al., "Yuan identification of TCM constitution and function of islet $\beta$ cells in patient with newly onset type 2 diabetes," Journal of Traditional Chinese Medicine, vol. 33, no. 9, pp. 48-50, 2018.

[28] N. Lang, "Study on the relationship between different constitution of traditional Chinese medicine and endothelial function in the elderly with diabetes mellitus," Journal of Clinical Medical Literature Electronic Edition, vol. 5, no. 89, p. 21, 2018.

[29] L. H. Jiang, Z. F. Wong, and X. Y. Hu, "The influence of prevention and treatment of integrated traditional Chinese and Western Medicine on the constitution of community diabetes patients," Chinese Journal of Information on Traditional Chinese Medicine, vol. 25, no. 2, pp. 20-25, 2018.

[30] L. P. Hu, D. H. Wang, and J. H. Li, "Correlation between socioeconomic status and TCM constitution in 200 patients with Type 2 diabetes mellitus," Hebei Journal of Traditional Chinese Medicine, vol. 40, no. 2, pp. 187-191, 2018.

[31] Y. H. Gao, A. L. Zhang, H. B. Yang et al., "Study on the constitution identification and syndrome differentiation of the elderly patients with diabetes and hypertension," Hebei Journal of Traditional Chinese Medicine, vol. 40, no. 11, pp. 46-49, 2018.

[32] W. Y. Chen, B. X. Cheng, and L. Q. Wang, "Observation on the effect of TCM Constitution Differentiation on community management of type 2 diabetes in the elderly," Modern Medicine and Health Research, vol. 2, no. 17, p. 144, 2018.

[33] D. Zhou, N. S. Lin, F. Wang et al., "Correlation analysis of Traditional Chinese medicine Constitution type and blood glucose control in type 2 diabetes patients," Health Education and Health Promotion, vol. 12, no. 3, pp. 200-203, 2017.

[34] J. Wang, "Relationship between lifestyle and Traditional Chinese medicine Constitution in patients with chronic disease diabetes," Chinese Practical Medicine, vol. 9, pp. 187-188, 2017.

[35] Y. Shen, H. Tang, and D. Zhou, "Research on distribution characteristics of TCM constitution of patients with type 2 diabetes mellitus and related risk factors," World Chinese Medicine, vol. 12, no. 10, pp. 2487-2491, 2017.

[36] B. Shen, C. Yu, W. Yu et al., "Investigation and analysis of TCM constitution of 333 patients with type 2 diabetes in beijing pinggu district," Chinese medicine Modern Distance Education of China, vol. 15, no. 16, pp. 58-60, 2017.

[37] J. L. Qiu, Effect of Characteristic Health Education Based on Constitution Recognition of Traditional Chinese Medicine on Metabolic Control of Diabetic Patients, Xinjiang Medical University, Wulumuqi, China, 2017.

[38] J. X. Lv, Study on the Relationship between the Constitution of Traditional Chinese Medicine and Risk Factors in Type 2 Diabetes Mellitus, Tianjin University of traditional Chinese Medicine, Tianjin, China, 2017.

[39] Y. Liu, H. Y. Zhu, and Q. S. Wu, "Study on the level of inflammatory factors in diabetic patients with different constitution of traditional Chinese Medicine," Heilongjiang Medicine and Pharmacy, vol. 40, no. 3, p. 121, 2017.

[40] X. Li, "Analysis of the effect of TCM Constitution identification on the treatment and intervention of elderly diabetes patients in the community," Health and Nutrition in China, vol. 27, no. 1, p. 383, 2017.

[41] G. C. Jing, W. J. Gu, and M. R. Zhang, "Study on relationship between constitution of TCM and personality characteristics of patients with type 2 diabetes mellitus," China Journal of Traditional Chinese Medicine and Pharmacy, vol. 32, no. 11, pp. 4946-4948, 2017. 
[42] Y. J. Jing, Correlation Analysis of Multiple Factors in Type 2 Diabetes Patients with Qi-Deficiency Constitution, Yunnan College of Traditional Chinese Medicine, Kunming, China, 2017.

[43] Q. Huang, Study on TCM Constitution Intervention of "2394" Chronic Disease Management Model in Type 2 Diabetes Patients, Southern Medical University, Guangzhou, China, 2017.

[44] Q. Huang, X. S. Zhao, S. N. Sun et al., "TCM constitutional types and cardiovascular risk factors on 367 type 2 diabetic patients," Chinese Archives of Traditional Chinese Medicine, vol. 35, no. 1, pp. 229-232, 2017.

[45] Q. Huang, W. R. Wang, J. F. Tan et al., "Observation of TCM constitutions in patients with different stroke risks in type 2 diabetes mellitus," Zhejiang Medical Education, vol. 16, no. 5, pp. 46-48, 2017.

[46] Y. B. Zhu, H. M. Shi, Q. Wang et al., "Association between nine types of TCM constitution and five chronic diseases: a correspondence analysis based on a sample of 2660 participants," Evidence-Based Complementary and Alternative Medicine, vol. 2017, Article ID 9439682, 7 pages, 2017.

[47] J. Feng, "Application of TCM Constitution identification in health management of community diabetes," Health Care Guide, vol. 5, pp. 46-48, 2017.

[48] J. X. Zou and W. P. Wang, "Investigation of TCM syndrome type and Constitution type in type 2 diabetes patients," Health Nutrition in China, vol. 26, no. 25, p. 149, 2016.

[49] L. Zhu, L. G. Liu, and L. Zhu, "Study on relationship between type 2 diabetes mellitus and constitution of inhabitants in community," Journal of Inhabitants in Community," Clinical And Experimental Medicine, vol. 26, no. 12, pp. 1169-1174, 2016.

[50] J. L. Xue, M. Z. Li, and Y. Z. Gong, "The characteristics of TCM constitution in type 2 diabetes patients in Baoshan District, Shanghai," Shanghai Preventive Medicine, vol. 28, no. 7, pp. 453-459, 2016.

[51] Y. M. Xu and D. Liu, "Investigation and Analysis on the constitution type of traditional Chinese medicine in the elderly patients with type 2 diabetes in the community," Shanxi Journal of Traditional Chinese Medicine, vol. 32, no. 2, pp. 45-46, 2016.

[52] H. J. Xie, G. F. Zhang, Y. J. Peng et al., "Investigation and analysis of TCM constitution characteristics of 426 elderly patients with type 2 diabetes in Zhengzhou city," Clinical Research of Traditional Chinese Medicine, vol. 8, no. 13, pp. 27-29, 2016.

[53] Z. W. Wang, J. Y. yang, B. Lei et al., "Effect of TCM constitution regulation on treatment effect and life quality of elder patients with type 2 diabetes mellitus," World Chinese Medicine, vol. 11, no. 8, pp. 1606-1609, 2016.

[54] K. F. Wang, Q. Yan, and C. M. Li, "Characteristics and correlation analysis of Traditional Chinese medicine constitution in 300 diabetic patients in Qitaihe Area," Diet Care, vol. 3 , no. 12, 2016.

[55] J. S. Wang, The Correlation Study of Efficacy of Exenatide or Saxapliptin in Type 2 Diabetes and Plasma GC, Chinese Medicine Body Constitution, Chengdu University of Traditional Chinese Medicine, Chengdu, China, 2016.

[56] Z. Z. Ren, H. Zhao, L. Yang et al., "Research on application of TCM constitution identification and individual health management guidance in type 2 diabetes of community," Guangming Journal of Chinese Medicine, vol. 31, no. 18, pp. 2603-2604, 2016.

[57] Z. Li, "A clinical analysis of TCM constitution distribution of hypertension and diabetes and treating different diseases by the same method," Clinical Journal of Chinese Medicine, vol. 8, no. 4, pp. 70-71, 2016

[58] J. Li, Y. Zhu, Y. S. Chen et al., "Distribution of TCM constitutions in 302 patients with type 2 diabetes in Kunming," Chinese Medicine Research, vol. 29, no. 1, pp. 11-13, 2016.

[59] W. B. Huang, Preliminary Research on Diabetic Patients' TCM Body Constitution Among Malaysian Chinese in Klang Valley, Chengdu University of Traditional Chinese Medicine, Chengdu, China, 2016.

[60] Y. K. Hua, W. Yuan, Y. P. Be et al., "Investigation and analysis of TCM constitution among 931 patients with diabetes in yangpu district of shanghai," Guiyang College of Traditional Chinese Medicine, vol. 38, no. 3, pp. 71-74, 2016.

[61] J. X. Zou and W. P. Wang, "Investigation on TCM syndrome type and constitution type of type 2 diabetes mellitus," China Health Nutrition, vol. 26, no. 25, p. 149, 2016.

[62] Z. Y. Zhao, The Correlation Research of Dangerous TCM Constitution and Type 2 Diabetes Mellitus in Inner Mongolia Region, Inner Mongolia Medical University, Neimongol, China, 2015.

[63] B. Zhang, "Discussion on the relationship between the onset of diabetes and the constitution of traditional Chinese Medicine," Biotech World, vol. 10, pp. 268-269, 2015.

[64] J. Yu, Traditional Chinese Medical Body Constitution, its Related Clinical Characteristics, and Diabetes-Associated Chronic Complications in Patients with Type 2 Diabetes, Dalian Medical University, Dalian, China, 2015.

[65] T. Yang, Studies on Building Platform of Type Diabetes Management in Qingdao Area Based on the Constitution Identification of Traditional Chinese Medicine, Qingdao University, Qingdao, China, 2015.

[66] X. Y. Xiang and J. Q. Qian, "Study on the relationship between TCM constitution characteristics and body mass index, fasting blood glucose in type 2 diabetes mellitus," AsiaPacific Traditional Medicine, vol. 11, no. 16, pp. 91-92, 2015.

[67] X. M. Wu and Z. H. Hao, "TCM constitution analysis of type 2 diabetic patients with different body mass index," Journal of Hubei University for Nationalities, vol. 32, no. 3, pp. 21-23, 2015.

[68] Q. L. Wu, S. R. Guo, J. H. Ke et al., "Clinical investigation on the constitution of diabetes mellitus patients in Jinjiang area," Journal of Clinical Medical Literature, vol. 2, no. 21, pp. 4486-4487, 2015.

[69] J. F. Wei, Q. Liu, and J. G. Chen, “Analysis on application effect of TCM constitution identification and intervention in the management of type 2 diabetes patients in community," Guiding Journal of Traditional Chinese Medicine and Pharmacy, vol. 21, no. 13, pp. 35-38, 2015.

[70] G. Z. Wang, J. L. Zeng, S. Zeng et al., "Investigation report on constitution condition of type 2 diabetes patients in Meizhou Hospital of traditional Chinese Medicine," Guiding Journal of Traditional Chinese Medicine and Pharmacy, vol. 21, no. 2, pp. 88-89, 2015.

[71] H. Lu, "Analysis on the constitution of diabetes mellitus patients over 60 years old in Anting Town," The World Clinical Medicine, vol. 9, no. 5, pp. 10-12, 2015.

[72] X. D. Liu, X. Q. Lv, and N. W. Liu, "Investigation on TCM syndrome types and TCM constitutions of II type diabetes," Shanxi Journal of Traditional Chinese Medicine, vol. 31, no. 1, pp. 41-42, 2015

[73] Z. S. Li, "Analysis of risk factors of type 2 diabetes base on TCM constitution differentiate," Journal of Taishan Medical College, vol. 36, no. 11, pp. 1218-1220, 2015. 
[74] J. H. Li, Analysis of the Constitution Characteristics of the Elderly Patients with Diabetes Mellitus in Dongfu Community, Xiamen, Fujian University of Traditional Chinese Medicine, Xiamen, China, 2015.

[75] G. R. Hu, L. Zhang, Y. H. Zeng et al., "The clinical investigation and Research on the constitution of diabetes in Nanchang area," Journal of Practical Diabetology, vol. 11, no. 1, pp. 33-34, 2015.

[76] L. J. Chen and J. Y. Zhou, Study on the Distribution of TCM Constitution of Type 2 Diabetes in Patients with Constitution Identification, Annual Meeting of Constitution of Traditional Chinese Medicine of Chinese Medical Association, Zhejiang, China, 2015.

[77] X. Y. Xiao, B. Y. Li, and R. Zhou, "Investigation and analysis of TCM Constitution of type 2 diabetes patients in Sanshui District," Global Traditional Chinese Medicine, vol. 8, no. S2, p. 163, 2015.

[78] L. Zhu, C. Y. Tian, Q. Lin et al., "Study on the constitution of traditional Chinese medicine of type 2 diabetes in Tangshan," Journal of Clinical Medical Literature Electronic Edition, vol. 1, no. 8, pp. 1419-1420, 2014.

[79] D. F. Yang and W. R. Wang, "General information," AsiaPacific Journal of Clinical Oncology, vol. 10, no. 2, pp. 49-50, 2014.

[80] X. Y. Xiang and Y. Z. Ran, "Investigation on constitution type and TCM clinical Syndrome type of 270 patients with type 2 diabetes," World Traditional Chinese Medicine, vol. 9, no. 12, pp. 1599-1602, 2014.

[81] X. Y. Xiang, Investigation on Constitution Type and TCM Clinical Syndrome Type of Patients with Type 2 Diabetes Mellitus in Liuhe District of Taicang City, Nanjing University of Chinese Medicine, Nanjing, China, 2014.

[82] H. Wang, X. H. Zhang, and H. F. Liu, "Study on the relationship between the Constitution and family heredity of type 2 diabetes," World Chinese Medicine, vol. 36, no. 3, pp. 201-204, 2014.

[83] Y. H. Li, J. Q. Yang, and X. L. Huang, "The application of constitution identification in "preventive treatment" health management of diabetes in community," Journal of Zhejiang Chinese Medical University, vol. 38, no. 4, pp. 120-122, 2014.

[84] J. Q. Chen, Y. D. Guo, Y. Q. Zhu et al., "Studies nanyang city in type 2 diabetes patients and their constitution characteristics and the relationship between TCM," China Journal of Chinese Medicine, vol. 29, pp. 338-340, 2014.

[85] X. H. Zheng and Z. Y. Jian, "Logistic regression analysis of the relationship between constitution fitness, impaired glucose regulation and diabetes mellitus in 1882 middle-aged and elderly people in Guangzhou community," International Journal of Traditional Chinese Medicine, vol. 35, no. 6, pp. 481-484, 2013.

[86] X. Q. Wu, Y. Y. Luo, and J. H. Xu, "Investigation of traditional Chinese medical constitution of type 2 diabetes mellitus patients and its relationship with insulin resistance and insulin secretion," Journal of Guangzhou University of Traditional Chinese Medicine, vol. 30, no. 3, pp. 312-315, 2013.

[87] L. L. Tang, Analysis of the Relationship between the Constitution Type of TCM and Sex, Age, Body Mass Index in Type 2 Diabetes Mellitus, Guangxi University of Traditional Chinese Medicine, Nanning, China, 2013.

[88] J. R. Ma, "A study on the relationship between biased constitution of traditional Chinese medicine and diabetes mellitus and impaired glucose regulation," Clinical Journal of Integrated Traditional Chinese and Western Medicine, vol. 13, no. 4, pp. 56-57, 2013.
[89] W. H. Liu, "Correlation analysis of TCM Constitution with obesity, blood lipid and hemagglutination index in type 2 diabetes," Guide of China Medicine, vol. 11, no. 8, pp. 624-625, 2013.

[90] L. F. Li, W. H. Tang, Y. Q. Wang et al., "Relationship between TCM constitution and B-cell function in patients with type 2 diabetes," Jilin Journal of Traditional Chinese Medicine, vol. 33, no. 1, pp. 45-47, 2013.

[91] J. N. Zhang, L. Zhang, and R. Luo, Analysis of Related Factors of TCM Constitution in Hong Kong Diabetes Patients, China Association of Chinese Medicine, Haikou, China, 2011.

[92] W. T. Han, A Study on the Constitution of TCM in Type 2 Diabetes Mellitus, Beijing University of traditional Chinese Medicine, Beijing, China, 2011.

[93] P. Han, X. Q. Bai, Y. M. Lou et al., "Analysis of the relationship between the biased constitution of traditional Chinese medicine and the impairment of diabetes and glucose regulation," Beijing Journal of Traditional Chinese Medicine, vol. 30, no. 9, pp. 656-658, 2011.

[94] J. N. Zhang, Study on the Constitution Characteristics of TCM in 534 Patients with Type 2 Diabetes Mellitus, Southern Medical University, Guangzhou, China, 2010.

[95] Y. Yan, P. Zhu, F. Zhang et al., "Cases of type 2 diabetes and related major," China Journal of Chinese Medicine, vol. 25, no. 6, pp. 1154-1156, 2010.

[96] G. Cui, A Survey of the Common Constitution Types of Diabetes Mellitus in Xianyang Area and its Health Management, Shanxi University of Traditional Chinese Medicine, Xianyang, China, 2009.

[97] M. Li, S. Mo, Y. Lv et al., "A study of traditional chinese medicine body constitution associated with overweight, obesity, and underweight," Evidence-Based Complementary and Alternative Medicine, vol. 2017, Article ID 7361896, 8 pages, 2017.

[98] China Association of Chinese Medicine, Classification and Determination of TCM Constitution, China Press of TCM, Beijing, China, 2009.

[99] L. Li, J. Feng, H. Yao et al., "Gene expression signatures for phlegm-dampness constitution of Chinese medicine," Science China Life Sciences, vol. 60, no. 1, pp. 105-107, 2017.

[100] X. Liang, Q. Wang, Z. Jiang et al., "Clinical research linking Traditional Chinese Medicine constitution types with diseases: a literature review of 1639 observational studies," Journal of Traditional Chinese Medicine=Chung I Tsa Chih Ying Wen pan, vol. 40, no. 4, pp. 690-702, 2020.

[101] Y. B. Zhu and Q. Wang, "Epidemiological investigation of constitution types of Chinese Medicine in general population: based on 21,948 epidemiological investigation date of nine provinces in China," Journal of Traditional Chinese Medicine and Pharmacy, vol. 24, no. 1, pp. 7-12, 2009.

[102] D. Zou, Y. Ye, N. Zou, and J. Yu, "Analysis of risk factors and their interactions in type 2 diabetes mellitus: a cross-sectional survey in Guilin, China," Journal of Diabetes Investigation, vol. 8, no. 2, pp. 188-194, 2017.

[103] H. Luo, L. Li, T. Li, X. Liao, and Q. Wang, "Association between metabolic syndrome and body constitution of traditional Chinese medicine: a systematic review and metaanalysis," Journal of Traditional Chinese Medical Sciences, vol. 7, no. 4, pp. 355-365, 2020.

[104] Y. L. Ma, H. Yao, W. J. Yang et al., "Correlation between traditional Chinese medicine constitution and dyslipidemia: a systematic review and meta-analysis," Evidence-Based Complementary and Alternative Medicine, vol. 2017, Article ID 1896746, 7 pages, 2017. 